\title{
Influência do Volume de Alongamento Estático dos Músculos Isquiotibiais nas Variavéis Isocinéticas
}

\author{
Influence of Static Stretching Volume in Isokinetic Variables \\ of Harmstrings
}

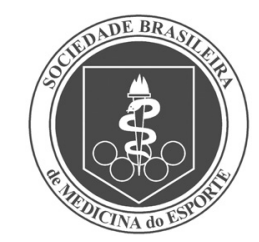

Artigo Original

Anselmo Grego Neto',

Elisangela Ferretti Manffra' ${ }^{1}$

1. Programa de Pós-graduação em Tecnologia em Saúde (PPGTS) da Pontifícia Universidade Católica do Paraná (PUCPR), Curitiba, Paraná, Brasil.

Endereço para correspondência: Elisangela Ferretti Manffra.

CCET - Curso de Engenharia Elétrica Rua Imaculada Conceição, 1.155,

Prado Velho 80215-980 Curitiba, PR. E-mail: elisangela.manffra@pucpr.br e elisangelaferretti@gmail.com

Submetido em 18/04/2007 Versão final recebida em 13/05/2008 Aceito em 06/08/2008

\section{RESUMO}

A realização de alongamento muscular antes de treinamentos e competições está enraizada na cultura dos profissionais que trabalham com prescrição de exercício. Acredita-se no alongamento como forma de aprimoramento do desempenho e prevenção de lesões. No entanto, muitos estudos têm mostrado que o alongamento pode produzir efeitos deletérios na capacidade de produção de força muscular. Algumas questões relevantes nesse contexto relacionam-se com o volume de alongamento necessário para produzir déficits de força e com os mecanismos fisiológicos responsáveis pelos mesmos. A proposta deste estudo foi investigar as alterações no desempenho isocinético do grupo muscular dos isquiotibiais mediante dois protocolos de alongamento estático com diferentes volumes. Trinta e seis voluntários adultos do sexo masculino foram distribuídos em três grupos: $E_{1}, E_{2}$ e $C$. Todos os participantes realizaram um aquecimento sistêmico e depois foram submetidos a avaliações da amplitude de movimento ativa (ADM) de flexão do quadril e isocinética. Aos participantes dos grupos $\mathrm{E}_{1}$ e $\mathrm{E}_{2}$ foram aplicados protocolos de alongamento estático com volumes de 180 s ( $4 \times 45$ s) e 360s ( $8 \times 45$ s), respectivamente, e estes foram novamente avaliados. Os participantes do grupo $C$ foram submetidos à segunda avaliação, após permanecer em repouso pelo tempo de 270s. As variáveis avaliadas foram a ADM, o pico de torque (PT), o trabalho máximo (TM) e o trabalho total (TT). Observou-se que ambos os protocolos promoveram aumento da ADM, mas as variáveis PT e TM sofreram déficits somente no grupo $E_{2}$. A variável TT, no entanto, manteve-se inalterada nos grupos $E_{1} e$ $E_{2}$. Os resultados sugerem, portanto, que as alterações na rigidez muscular, que causaram ganhos na ADM, não seriam as únicas responsáveis pelos déficits de força. Além disso, conclui-se que a capacidade máxima de produção de força é dependente do volume de alongamento, mas a produção de trabalho ao longo de algumas repetições não é.

Palavras-chave: força muscular, torque, amplitude de movimento, exercícios de alongamento muscular, dinamômetro.

\section{ABSTRACT}

Stretching exercises are commonly prescribed before training sessions and competitions aiming at performance improvement and reduction of injury risk. However, many studies have shown that muscular torque production capacity may be reduced just after stretching. Therefore, the stretching duration necessary to produce these acute force deficits, as well as the physiological mechanisms responsible for them are relevant issues. The aim of this work was to investigate the acute effects of static stretching protocols with different durations on the isokinetic hamstrings performance. Thirty-six young male volunteers took part in this study and were evenly distributed in three groups: $E_{1}, E_{2}$ and C. All of them performed a systemic warm-up for five minutes and went through active range of motion (AROM) of hip flexion and isokinetic assessment. The participants of groups $E_{1}$ and $E_{2}$ performed static stretching protocols of $180 \mathrm{~s}(4 \times 45 \mathrm{~s})$ and $360 \mathrm{~s}(8 \times 45 \mathrm{~s})$ respectively, and were evaluated again. The participants of group $C$ (control) remained at rest for a period of 270 s and were evaluated again. The variables considered were AROM, peak torque, maximum work and total work. Both stretching protocols were able to produce increase in AROM; however, only the longest protocol produced deficits on peak torque and maximum work. Total work was not affected by any of the stretching protocols, though. Therefore, these results suggest that changes in muscular stiffness, that caused AROM gain, would not be responsible alone for the force deficits. Moreover, one can conclude that the maximum muscular strength depends on the stretching duration, but the muscular work along some repetitions of an exercise does not.

Keywords: muscle strength, torque, muscle strength dynamometer, range of motion, muscle stretching exercises 


\section{INTRODUÇÃO}

Séries de alongamento muscular são rotineiramente incluídas em programas de exercícios para atletas sob a prerrogativa de que elas previnam eventuais lesões e otimizem o desempenho desportivo(1). Assim, a realização de exercícios de alongamento durante o aquecimento tornou-se prática corrente na preparação de atletas antes dos treinamentos e das competições ${ }^{(24)}$.

Mais especificamente, a técnica de alongamento estático tem sido utilizada por ser considerada bastante eficiente para produzir aumento agudo na amplitude de movimento articular ${ }^{(5,6)}$. Por esse motivo, inúmeros profissionais prescrevem exercícios de alongamento por meio dessa técnica durante o trabalho de aquecimento ${ }^{(7)}$. Entretanto, atualmente, muitos estudos têm apontado que a técnica estática pode induzir perda aguda da ordem de 5 a 30\% da força máxima e da potência de grupos musculares previamente alongados ${ }^{(2,5,7,8)}$. Esses achados têm levado alguns pesquisadores a questionarem a inclusão de exercícios de alongamento durante o período de aquecimento, sobretudo antes de atividades que exijam força e potência musculares, especialmente em provas de curta duração(7,8).

Duas hipóteses primárias têm sido propostas para explicar o chamado déficit transitório na força induzido pelo alongamento ${ }^{(8,9)}$. A primeira delas refere-se a alterações da rigidez muscular e a outra à alteração na ativação muscular em resposta ao exercício de alongamento ${ }^{(8)}$. Um dos estudos relevantes sobre este tema é trabalho de Marek et al. ${ }^{(8)}$. em que foram estudados os efeitos da aplicação das técnicas de alongamento estático e de facilitação neuromuscular proprioceptiva (FNP) sobre o desempenho isocinético da musculatura quadricipital. Os principais achados do estudo de Marek et al. ${ }^{(8)}$ foram déficits pós-alongamento no pico de torque, na potência média e na amplitude do sinal eletromiográfico para ambos os protocolos de alongamento. Esses resultados demonstram, portanto, que as duas técnicas de alongamento foram capazes de alterar tanto a relação comprimento-tensão da unidade musculotendínea quanto o nível de ativação muscular. Dessa forma, observa-se que a técnica adotada talvez não seja um fator tão relevante para o déficit de força causado pelo alongamento.

Comparando diversos estudos, observa-se que o volume dos protocolos de alongamento, este sim é um fator relevante na perda de força ${ }^{(2,7)}$. Assim como Marek et al. ${ }^{(8)}$, os resultados de Kokkonen et al. ${ }^{(7)}$ demonstram que protocolos com duração de 15, 16 e 20 minutos reduzem a capacidade de produção de força da musculatura de quadríceps. No estudo de Zakas et al. ${ }^{(2)}$, investigou-se o efeito do alongamento estático nessa mesma musculatura e observou-se que protocolos de cinco e oito minutos causaram déficit significativo no torque isocinético, mas nenhuma diferença significativa foi constatada para um protocolo de 30 segundos. Já Brandenburg ${ }^{(10)}$ encontrou déficits significativos na performance isocinética dos isquiotibiais após protocolos de alongamento estático com volumes de apenas 15 e 30 segundos. Sendo assim, a questão de qual seria tempo de permanência na postura de alongamento capaz de produzir o déficit de força ainda é um aspecto bastante controverso na literatura.

Considerando os achados citados no parágrafo anterior, poderse-ia especular se esse tempo é dependente da musculatura alongada em decorrência da própria conformidade muscular(8) ou do tipo predominante de suas fibras musculares ${ }^{(2,7,9)}$. Chama a atenção o fato de a maioria dos trabalhos que abordam o déficit de força induzido pelo alongamento ter se concentrado no grupo muscular do quadríceps ${ }^{(5,7,8)}$. Porém, considerando a realidade da prática esportiva, o grupo muscular dos isquiotibiais tem também um papel bastante importante ${ }^{(12)}$. Muitas vezes, esse grupo é responsável pelo controle excêntrico dos movimentos da articulação do joelho, como nas mudanças bruscas de direção, presentes em vários esportes ${ }^{(4)}$. Além disso, os isquiotibiais trabalham com o quadríceps em um sistema agonistaantagonista e, em termos de capacidade de geração de força, os dois grupos devem estar equilibrados para que os riscos de lesões sejam minimizados ${ }^{(4,13)}$.

Dentro desse contexto, a proposta deste trabalho foi estudar a influência do volume do alongamento estático dos isquiotibiais em seu desempenho isocinético. Foram comparados os efeitos de dois protocolos de alongamento estático com volumes de três e seis minutos realizados empregando-se uma postura de auto-alongamento com objetivo de simular realidade do emprego dos exercícios de alongamento, tanto nos protocolos de aquecimento de atletas como na reabilitação.

\section{MÉTODOS}

A presente pesquisa teve aprovação do Comitê de Ética em Pesquisa com Seres Humanos da PUCPR, sob o número de registro 902. Participaram do estudo 36 indivíduos do sexo masculino, hígidos, fisicamente ativos, com média de idade de 19 ( \pm 2 ) anos, massa de 63,8 $( \pm 5,3) \mathrm{kg}$ e estatura de $173( \pm 4,7) \mathrm{cm}$, todos com o membro inferior direito dominante. Todos os participantes assinaram um termo de consentimento livre e esclarecido antes de tomar parte do estudo. Os participantes foram alocados para um dos três grupos por meio de sorteio simples, logo após a anamnese. À medida que um dos grupos foi completado, este foi excluído do sorteio. O mesmo procedimento foi realizado com o segundo grupo até que ficasse completo, sendo os participantes remanescentes automaticamente alocados para o terceiro grupo. Estabelecidos os grupos, os participantes dos grupos estático $1\left(E_{1}\right)$ e estático $2\left(E_{2}\right)$ foram submetidos a protocolos de alongamento com volumes de 180 e 360 segundos, respectivamente. Os participantes do grupo controle (C) não realizaram nenhum tipo de alongamento.

Para mensuração da massa corporal dos sujeitos foi empregada uma balança mecânica (Techline, modelo AAS 320), com precisão estimada de 0,3kg. A mensuração da estatura dos sujeitos foi realizada por meio de um estadiômetro (Standard ES - 2030).

Para a avaliação do desempenho muscular foi empregado o dinamômetro isocinético (Cybex, modelo NORM 7000), módulo de membros inferiores. Um flexímetro (Flexitest, modelo 1496) foi empregado para as avaliações da amplitude de movimento da articulação do quadril.

Os participantes realizaram um aquecimento sistêmico em bicicleta ergométrica (Biocycle 2600 Eletromagnetic da marca Moviment) com carga livre $(0,5 \mathrm{kpm})$ durante cinco minutos ${ }^{(8)}$. Após o aquecimento, os participantes foram submetidos a uma avaliação da amplitude de movimento (ADM) em relação à flexão do quadril, seguida de uma avaliação isocinética inicial (pré-alongamento). Em seguida, os participantes realizaram seus respectivos protocolos de alongamento, à exceção do grupo controle, que permaneceu em repouso durante o tempo médio correspondente à aplicação dos protocolos de alongamento (270 segundos). Concluída essa etapa, foi realizada uma nova avaliação da ADM de flexão do quadril e isocinética (pós-alongamento) seguindo os mesmos parâmetros das avaliações iniciais. 
Para avaliação da ADM, o flexímetro era posicionado na face lateral da coxa direita, com o voluntário em decúbito dorsal(14). Em seguida, o sujeito realizava ativamente o movimento de flexão da articulação do quadril até sua amplitude máxima com o joelho plenamente estendido e o tornozelo em posição neutra; o valor do ângulo (em graus) apontado pelo flexímetro era registrado sempre pelo mesmo avaliador. Esse procedimento foi repetido três vezes e a média das três mensurações foi o valor considerado no estudo. A posição do flexímetro era demarcada com uma caneta dermatográfica para que o mesmo pudesse ser colocado precisamente na mesma posição na avaliação da ADM pós-alongamento. Todas as medidas de flexibilidade foram realizadas pelo mesmo avaliador.

A fim de estimar a reprodutibilidade da medida da ADM, foi calculada sua variabilidade percentual média, seguindo-se o seguinte procedimento: para cada um dos valores de ADM calculou-se a variabilidade como sendo a razão entre o desvio padrão e a média das três mensurações. Tomando-se o valor médio como referência (100\%), calculou-se a variabilidade percentual para cada medida. Calculou-se, então, a média das variabilidades percentuais de todas as medidas, obtendo-se o valor de 4,0\%.

Para as avaliações isocinéticas os voluntários eram posicionados sentados, com a cadeira do dinamômetro posicionada com $85^{\circ}$ de flexão do quadril. 0 eixo mecânico do aparelho era alinhado com o epicôndilo lateral do fêmur, a referência óssea para o eixo biológico da articulação do joelho. Os voluntários foram presos firmemente à cadeira do dinamômetro com cintos na região do tronco, do quadril e da coxa, a fim de evitar compensações indesejáveis. A amplitude de movimento foi limitada entre $105^{\circ}$ de flexão e $0^{\circ}$ de extensão do joelho. Antes da realização de cada avaliação isocinética foi realizada a correção para a gravidade como parte integrante dos procedimentos de preparo do dinamômetro isocinético ${ }^{(2,7,10)}$. As avaliações foram realizadas em relação ao movimento de flexão do joelho, no modo concêntrico e ocorreram à velocidade angular de $60^{\circ} \cdot \mathrm{s}^{-1}$. Durante a avaliação isocinética inicial os sujeitos realizaram três repetições submáximas com fins de familiarização com o dinamômetro, seguidas de um intervalo de um minuto, ao término do qual os sujeitos executaram as três repetições máximas, cujos dados foram registrados. Na avaliação isocinética final, não havendo mais a necessidade da série de familiarização, os voluntários executaram somente as três repetições máximas, também concêntricas, na velocidade de $60^{\circ} \cdot \mathrm{s}^{-1}$. Todos os sujeitos tiveram encorajamento verbal do mesmo avaliador durante os testes isocinéticos. Todos os dados isocinéticos considerados no presente estudo são relativos ao membro dominante dos sujeitos avaliados.

Os sujeitos dos grupos $E_{1}$ e $E_{2}$ foram submetidos à técnica de alongamento estático aplicada no grupo muscular dos isquiotibiais sob supervisão direta do pesquisador. A postura escolhida para aplicação do alongamento foi a chamada de "sentar e alcançar", considerada eficiente para o alongamento dos músculos isquiotibiais ${ }^{(6)}$.

Para a aplicação dos protocolos, os sujeitos foram instruídos a permanecer sentados em uma maca com os joelhos em extensão total, os tornozelos livres, em posição neutra, e realizar flexão máxima do tronco com os membros superiores em extensão. Após atingir a ADM final na qual deveriam permanecer para realizar o alongamento, os sujeitos eram inquiridos a respeito do nível de desconforto subjetivo provocado pelo exercício, sendo então informados de que esse deveria ser o máximo tolerável, sem, no entanto, provocar dor. Por se tratar de uma postura de autoalongamento, os exercícios foram realizados sem interferência direta do pesquisador. Porém, os sujeitos eram permanente e veementemente encorajados a executar os exercícios na máxima amplitude articular possível.

Para coibir compensações, uma tira de velcro era posicionada no terço distal da coxa dos sujeitos, de forma a evitar a flexão dos joelhos. $O$ protocolo de alongamento para o grupo $E_{1}$ era composto por quatro séries de 45 segundos cada uma, com intervalo de 60 segundos entre elas, perfazendo um total de 180 segundos de permanência efetiva do sujeito na postura de alongamento. Para o grupo $\mathrm{E}_{2}$, o protocolo era composto por oito séries de 45 segundos, também sendo permitidos intervalos de 60 segundos entre elas, perfazendo um total de 360 segundos na postura de alongamento.

O tempo decorrido entre o término da última repetição do protocolo de alongamento e a avaliação pós-alongamento da ADM de flexão do quadril era de aproximadamente 80 segundos. Esse tempo permite observar os efeitos agudos do alongamento estático, tendo em vista que trabalhos na literatura demonstram que efeitos perduram de três ${ }^{(11)}$ até 10 minutos $^{(8)}$.

As variáveis isocinéticas avaliadas foram o pico de torque (PT), o trabalho máximo (TM) e o trabalho total (TT). Em relação às variáveis analisadas, cabe ressaltar que o PT e o TM são extraídos da melhor curva de torque que o sujeito produziu, enquanto que o TT corresponde à soma do trabalho muscular exercido nas três repetições executadas. Tais valores foram obtidos diretamente do arquivo fornecido pelo dinamômetro.

Para a análise estatística, avaliou-se inicialmente a condição de normalidade dos dados pelo teste de Kolmogorov-Smirnov e a homogeneidade das variâncias pelo teste de Levène. Para a comparação dos três grupos em relação às variáveis antropométricas e às variáveis pré-alongamento foi usada a análise de variância com um fator (ANOVA). Para a comparação entre avaliações pré e pós-alongamento, dentro de cada grupo, foi usado o teste $t$ de Student para amostras pareadas $^{(15)}$.

Para todos os membros de cada grupo, foram determinadas as diferenças percentuais entre os valores pré e pós-alongamento de cada variável(15), sendo:

Diferença percentual $=100\left(\right.$ valor $_{\text {pós }}-$ valor $\left._{\text {pré }}\right)$ /valor $_{\text {pré }}$

Os três grupos foram comparados em relação às diferenças percentuais através da análise de covariância (ANCOVA), tendo os valores pré-alongamento como covariável ${ }^{(16)}$. Nos casos em que houve diferença entre os grupos, foram realizadas comparações múltiplas entre os grupos utilizando o teste LSD.

Em todos os testes, valores de $p<0,05$ indicaram significância estatística.

O software utilizado para a realização do tratamento estatístico do presente trabalho foi o SPSS (versão 14).

\section{RESULTADOS}

As características antropométricas dos três grupos estão resumidas na tabela 1, onde é possível observar a homogeneidade entre os mesmos.

Na tabela 2 são apresentados os valores absolutos médios e seus respectivos desvios padrões encontrados nas avaliações iniciais e finais de ADM e isocinética dos músculos isquiotibiais em relação às variáveis investigadas para cada grupo individualmente (intragrupo). É importante reportar que o teste ANOVA não revelou diferenças significativas entre os valores das avaliações iniciais dos três grupos. 
Tabela 1. Médias dos dados antropométricos dos grupos

\begin{tabular}{c|c|c|c|c}
\hline Variável & Grupo $\mathrm{E}_{\mathbf{1}}$ & Grupo $\mathrm{E}_{\mathbf{2}}$ & Controle & Valor de $\mathbf{p}^{*}$ \\
\hline Idade (anos) & $21,1 \pm 2,7$ & $20,9 \pm 3,0$ & $21,8 \pm 3,5$ & 0,743 \\
\hline Massa $(\mathrm{kg})$ & $66,7 \pm 12,4$ & $67,3 \pm 10,3$ & $68,8 \pm 6,0$ & 0,874 \\
\hline Estatura $(\mathrm{cm})$ & $173,3 \pm 7,5$ & $176,0 \pm 6,5$ & $177,0 \pm 5,5$ & 0,378 \\
\hline IMC $\left(\mathrm{kg} / \mathrm{m}^{2}\right)$ & $22,1 \pm 2,7$ & $21,7 \pm 2,2$ & $22,0 \pm 2,0$ & 0,905 \\
\hline
\end{tabular}

$\left({ }^{*}\right)$ Valores de $p$ calculados pelo teste ANOVA com um fator utilizado na comparação entre os três grupos, $p \leq 0,05$ indica diferença entre os grupos.

Tabela 2. Valores das médias absolutas das avaliações pré e pós-teste das variáveis para cada grupo

\begin{tabular}{|c|c|c|c|c|}
\hline \multicolumn{2}{|c|}{ Variável } & \multirow{2}{*}{$\begin{array}{c}\text { Grupo } \mathrm{E}_{1} \\
78,1 \pm 8,1\end{array}$} & \multirow{2}{*}{$\begin{array}{c}\text { Grupo } \mathbf{E}_{\mathbf{2}} \\
83,8 \pm 12,7\end{array}$} & \multirow{2}{*}{$\begin{array}{c}\text { Controle } \\
79,2 \pm 14,8\end{array}$} \\
\hline \multirow{3}{*}{ ADM (graus) } & Pré & & & \\
\hline & Pós & $82,1 \pm 10,4$ & $89,0 \pm 11,7$ & $78,5 \pm 13,1$ \\
\hline & Valor de $\mathrm{p}^{*}$ & 0,008 & $<0,001$ & 0,494 \\
\hline \multirow{3}{*}{$\begin{array}{l}\text { Pico de torque } \\
\text { (N.m) }\end{array}$} & Pré & $117,0 \pm 24,2$ & $121,3 \pm 15,0$ & $115,7 \pm 16,2$ \\
\hline & Pós & $116,9 \pm 25,2$ & $114,9 \pm 13,4$ & $118,7 \pm 19,2$ \\
\hline & Valor de $\mathrm{p}^{*}$ & 0,969 & 0,029 & 0,153 \\
\hline \multirow{3}{*}{$\begin{array}{l}\text { Trabalho máximo } \\
\text { (J) }\end{array}$} & Pré & $154,8 \pm 33,1$ & $162,2 \pm 21,7$ & $149,6 \pm 25,7$ \\
\hline & Pós & $154,2 \pm 36,2$ & $154,4 \pm 22,4$ & $155,9 \pm 28,2$ \\
\hline & Valor de $\mathrm{p}^{*}$ & 0,825 & 0,017 & 0,009 \\
\hline \multirow{3}{*}{ Trabalho total (J) } & Pré & $446,6 \pm 95,5$ & $464,8 \pm 66,4$ & $434,5 \pm 77,4$ \\
\hline & Pós & $438,3 \pm 105,8$ & $451,9 \pm 70,3$ & $446,3 \pm 79,7$ \\
\hline & Valor de $\mathrm{p}^{*}$ & 0,374 & 0,190 & 0,036 \\
\hline
\end{tabular}

$\left({ }^{*}\right)$ Valor de $\mathrm{p}$ correspondente ao teste $\mathrm{t}$ de Student para comparação entre os valores pré e pós-alongamento dentro de cada grupo; $p \leq 0,05$ indica diferença entre as avaliações.

Em relação à variável amplitude de movimento (ADM) de flexão do quadril, a tabela 2 mostra que ambos os grupos de estudo $\left(E_{1}\right.$ e $E_{2}$ ) apresentaram aumentos significativos após o alongamento, enquanto que para o grupo controle (C) esta manteve-se inalterada, dentro da significância adotada ( $p \leq 0,05)$. Em relação à variável PT, apenas o grupo $E_{2}$ apresentou déficit significativo no teste pós-alongamento, sem que o mesmo comportamento tenha ocorrido com os grupos $E_{1}$ e $C$ para essa variável. Em relação à variável TM, tanto o grupo $E_{2}$ quanto o $C$ apresentaram variações significativas; no entanto, a variação ocorrida no grupo $E_{2}$ foi negativa (queda no TM), enquanto a do grupo C foi positiva (aumento do TM). Os dados do grupo $E_{1}$ mantiveram-se inalterados para essa variável. Em relação ao TT, nenhum dos grupos de estudo $\left(E_{1}\right.$ e $\left.E_{2}\right)$ apresentou variações significativas, diferentemente do grupo $C$, em que se observou aumento dessa variável.

Na tabela 3 são mostradas as diferenças percentuais obtidas entre condições pré e pós-alongamento para os três grupos, bem como os valores de $p$ resultantes da comparação entre os três grupos (intergrupos). As diferenças percentuais médias nas variáveis ADM, PT e TM mostraram-se heterogêneas entre os grupos, ao contrário da variável TT.
Tabela 3. Comparação das médias das diferenças percentuais entre os valores das avaliações pré e pós-teste entre os grupos

\begin{tabular}{l|c|c|c|c}
\hline \multicolumn{1}{c|}{ Variável } & Grupo $\mathrm{E}_{\mathbf{1}}$ & Grupo $\mathrm{E}_{\mathbf{2}}$ & Controle & Valor de $\mathbf{p}^{*}$ \\
\hline ADM & $5,0 \pm 4,9$ & $6,6 \pm 4,5$ & $-0,4 \pm 5,4$ & 0,001 \\
\hline Pico de torque & $-0,1 \pm 7,0$ & $-4,9 \pm 7,2$ & $2,5 \pm 5,4$ & 0,037 \\
\hline Trabalho máximo & $-0,8 \pm 8,2$ & $-4,8 \pm 6,0$ & $4,2 \pm 4,3$ & 0,006 \\
\hline Trabalho total & $-2,2 \pm 7,1$ & $-2,7 \pm 6,93$ & $2,9 \pm 3,9$ & 0,063 \\
\hline
\end{tabular}

$\left(^{*}\right)$ Valores de $p$ calculados pelo teste ANCOVA utilizado na comparação entre os três grupos; $p \leq 0,05$ indica diferença entre os grupos.

Observa-se, na tabela 2, que ambos os protocolos foram capazes de provocar aumento na ADM, porém, com valores percentuais ligeiramente diferentes, como mostra a tabela 3. A fim de checar a relevância desta diferença, foi aplicado o teste estatístico LSD para comparação entre os grupos $E_{1}$ e $E_{2}$ (sem a inclusão do grupo C), o qual não revelou diferença com relação à variação percentual da ADM nos dois grupos $(p=0,439)$.

\section{DISCUSSÃO}

Os dados da tabela 2 para a ADM de flexão do quadril demonstram que ambos os protocolos foram capazes de produzir aumento da mesma, concordando com outros estudos em que protocolos de alongamento da mesma técnica, porém com diferentes durações, foram eficazes em provocar aumento na ADM relacionada com a musculatura alongada ${ }^{(3,17)}$. É importante ressaltar que, apesar de a técnica de alongamento aqui empregada não ser específica para o grupo muscular dos isquiotibiais, a técnica de avaliação empregada pré e pós-alongamento era, sim, específica para esse grupo, o que permite inferir a influência dos protocolos administrados na ADM.

Ainda com relação à $A D M$, outro ponto a ser considerado é que as variações percentuais das $A D M s$ dos dois grupos de estudo $\left(E_{1}\right.$ e $\left.E_{2}\right)$ mostraram-se semelhantes dentro da significância estatística adotada. Isso leva a crer, num primeiro momento, que os dois protocolos teriam provocado o mesmo efeito sobre a ADM. Porém, esse resultado deve ser discutido com cuidado, porque remete à interpretação de que a rigidez da unidade musculotendínea (UMT) teria sido afetada da mesma forma pelos dois protocolos. Esse fato pode ser verdadeiro se houver um limiar a partir do qual as propriedades mecânicas da UMT passam a responder de forma independente do tempo de alongamento. Porém, essa discussão fica dificultada, por dois motivos. Primeiramente, por uma limitação no método de medida adotado que apresentou reprodutibilidade de 4\%, que é próxima dos valores das diferenças percentuais encontradas na tabela 3. Em segundo lugar, pelo fato de que a literatura geralmente não considera as variações percentuais, tal como foi feito na tabela 3, fornecendo apenas as medidas da ADM separadamente nas condições pré e pósalongamento $(2,3,7,8)$.

Ao contrário da ADM, as variáveis isocinéticas PT e TM comportaram-se de forma diferente nos grupos $E_{1}$ e $E_{2}$. Para essas variáveis, fica bastante clara a influência negativa que o maior tempo de duração do protocolo $E_{2}$ exerceu, provocando déficits significativos no desempenho isocinético dos músculos isquiotibiais, diferentemente do protocolo $E_{1}$. Esse dado é sugestivo de que as diferenças encontradas entre os grupos $E_{1}$ e $E_{2}$ em relação ao PT e ao TM possam ser atribuídas a outros fatores biomecânicos e/ou neurais que não 
alterações na rigidez muscular. De fato, no trabalho de Marek et al. ${ }^{(8)}$, encontrou-se diminuição na atividade eletromiográfica, sugerindo diminuição da ativação muscular em decorrência do alongamento, um mecanismo de redução de força não relacionado diretamente com a rigidez muscular.

Por outro lado, esse resultado também aponta para o fato de que a mensuração da ADM como uma medida indireta de estimação do nível de rigidez da UMT não seja um parâmetro consistente, conforme sugerem os achados de Brandenburg (10). Ainda nessa linha, Behm et al. ${ }^{(9)}$ citam que o nível de flexibilidade dos sujeitos não está diretamente relacionado com a magnitude dos déficits na força induzidos pelo alongamento. Dessa forma, a fim de elucidar melhor a influência do volume do alongamento nas propriedades viscoelásticas da UMT, seriam necessários estudos mais específicos para esse fim, tais como os que investigam os efeitos do alongamento na rigidez passiva ${ }^{(18)}$. Nessa situação, os elementos elásticos são, de certa forma, isolados dos elementos contráteis da UMT(18).

De qualquer forma, as diferenças de comportamento encontradas entre os grupos de estudo com relação aos déficits no pico de torque reforçam a hipótese, citada por outros autores, de que os déficits no desempenho muscular são maiores quanto maior a duração dos exercícios de alongamento ${ }^{(2,5,7,8,13)}$.

Embora a relação entre os efeitos agudos do alongamento e o desempenho de força seja muito mais debatida na literatura por meio da variável PT, o comportamento dos grupos em relação ao TM, apresentado no presente estudo, merece especial atenção. Isso por descrever o comportamento muscular de forma mais ampla que o PT, tendo em vista que o TM corresponde à área sob a curva de torque ao alongo de toda a amplitude de movimento da melhor repetição realizada pelo sujeito. Nesse sentido, este trabalho traz uma complementação ao estudo de Marek et al. ${ }^{(8)}$ que, segundo os próprios autores, não permitiu esclarecer como o alongamento poderia alterar a área sob a curva ao longo da amplitude total de movimento, pois não considerou toda a faixa de movimento no cálculo da potência média. Observou-se aqui que o TM comportou-se de forma semeIhante ao PT, apresentando déficit na avaliação pós-alongamento do grupo $E_{2}$, mas não do grupo $E_{1}$.O comportamento semelhante de PT e TM, observado neste estudo, permite inferir que, seja qual for o mecanismo que causa alteração na força, ele age ao longo de toda a curva comprimento-tensão.

Diante dos dados obtidos em relação às duas variáveis máximas (PT e TM), infere-se que dois fatores relacionados ao tempo de aplicação dos protocolos tenham influenciado nos resultados encontrados. O primeiro deles, de natureza mecânica, na medida em que a aplicação de protocolos de alongamento estático pode promover alterações nas propriedades viscoelásticas da UMT, ocasionando redução aguda em sua rigidez e alterando a relação comprimentotensão ideal das fibras musculares, mecanismo esse extensivamente descrito na literatura em trabalhos homólogos ${ }^{(8,9,12,13)}$. Outro aspecto que, infere-se, seja relevante para a compreensão do comportamento das variáveis PT e TM no presente estudo diz respeito à influência dos fatores neurais. Nesse sentido, considerando que os sujeitos de ambos os grupos de estudo $\left(E_{1}\right.$ e $\left.E_{2}\right)$ permaneciam nas posturas de alongamento respeitando o limite individual de desconforto máximo tolerável, infere-se que tenha havido a ativação do reflexo inibitório gerado pelos OTGs (inibição autogênica) em resposta ao estímulo provocado pelo alongamento dos músculos isquiotibiais $^{(11,12)}$.
O fenômeno da inibição autogênica foi extensivamente descrito por trabalhos, alguns dos quais realizaram avaliações eletromiográficas de superfície e constataram que tal fenômeno é responsável por redução significativa no número de unidades motoras recrutadas no grupo muscular sob a sua influência, limitando consideravelmente a produção de força por esses músculos ${ }^{(8,10,19)}$.

Infere-se que a referida influência dos fatores neurais tenha realmente acontecido neste estudo, tendo em vista que, ao contrário de outros nos quais a reavaliação de força era realizada com até 10 minutos de intervalo após o alongamento ${ }^{(7,8)}$, neste trabalho a avaliação pós-alongamento era realizada em cerca de 80s após o término de aplicação do estímulo. Porém, a falta de uma análise do sinal eletromiográfico não permite avaliar exatamente a influência dos fatores neurais neste trabalho. Pretende-se realizar essa análise em estudos futuros e examinar, inclusive, por quanto tempo ela pode persistir após o alongamento.

Ainda sobre os fatores neurais, Kokkonen et al. ${ }^{(7)}$ reiteram que a influência do reflexo de inibição autogênica é limitada pela duração do protocolo de alongamento. E levando-se em consideração o elevado limiar de excitabilidade do OTG (comparado com o fuso muscular) em relação ao alongamento estático, infere-se que o protocolo $E_{2}$ tenha desencadeado uma resposta mais contundente do OTG em comparação com o protocolo $E_{1}$, fato que se refletiu mais nos déficits do grupo $E_{2}$, ao contrário do grupo $E_{1}$.

Cabe aqui uma discussão sobre o comportamento do grupo controle, que não apresentou alteração significativa no PT, porém, teve aumento relevante no TM. Tal aumento pode ser atribuído a um provável efeito de treinamento e aprendizagem motora proporcionados pela avaliação isocinética inicial(19), que repercutiu positivamente tanto no desempenho do TM quanto no do TT.

No que se refere à variável trabalho total (TT), ao contrário do PT e do TM, este apresentou comportamento similar nos grupos $E_{1}$ e $E_{2}$, sem déficit perceptível pós-alongamento. Lembrando que o TT reflete a soma do trabalho realizado nas três repetições, essa é a única variável que traz consigo informações sobre as duas outras repetições que não a máxima. Esse comportamento dos grupos $E_{1}$ e $E_{2}$ sugere que tenha havido dissipação dos efeitos do alongamento no decorrer da execução das três repetições. Em outras palavras, infere-se que tenha havido recrutamento progressivo de unidades motoras no transcorrer da realização das três repetições capaz de sobrepujar os efeitos deletérios do alongamento, principalmente em relação ao grupo $E_{2}^{(17)}$. No entanto, para afirmar que esse recrutamento progressivo realmente ocorreu, seria necessário analisar separadamente a forma de cada uma das três curvas de torque obtidas levando em conta sua ordem temporal, o que não foi realizado neste estudo, mas deve ser feito em trabalhos futuros.

Os resultados deste trabalho sugerem que, para a musculatura de isquiotibiais, protocolos de alongamento preparatórios com volumes totais iguais ou superiores a 360 segundos podem comprometer o desempenho de atletas de alto rendimento, cujo sucesso seja dependente da capacidade muscular de produzir força máxima. 0 mesmo não acontece no caso de atletas cuja performance esportiva exija resistência muscular, pois, como indicam os resultados do presente estudo, parece haver dissipação dos efeitos do alongamento ao longo do continuum das contrações musculares. Uma vez que o protocolo de alongamento de 180 segundos, aqui investigado, não prejudicou as valências físicas de força e trabalho muscular, podese considerar que o mesmo possa ser executado mesmo antes de 
atividades que exijam a realização de força, seja em treinamento desportivo ou em reabilitação. Entretanto, vale ressaltar que é necessário ter cautela ao discutir as implicações práticas dos resultados científicos de único estudo como este, uma vez que foi realizado com uma amostra restrita, composta exclusivamente por indivíduos hígidos do sexo masculino compreendidos em uma estreita faixa etária (17-21 anos).

\section{CONCLUSÃO}

O desempenho isocinético dos isquiotibiais foi avaliado, neste trabalho, com auxílio das variáveis pico de torque (PT), trabalho máximo (TM) e trabalho total (TT). As variáveis máximas (PT e TM) mostraram-se sensíveis ao tempo de permanência dos voluntários na postura de autoalongamento: ambas apresentaram déficits após a aplicação do protocolo de seis minutos (8 X 45s), mas não sofreram variações após a aplicação do protocolo de três minutos (4 X 45s). A variável TT permaneceu inalterada após a aplicação de ambos os protocolos.

Pode-se concluir, então, que a capacidade máxima de produção de força do grupo muscular dos isquiotibiais dos voluntários deste estudo foi alterada pelo volume do alongamento (variáveis PT e TM). No entanto, quando se considera a produção de trabalho ao longo de algumas repetições (variável TT), não foi possível distinguir o efeito dos dois protocolos, mesmo um tendo o dobro da duração do outro.

Todos os autores declararam não haver qualquer potencial conflito de interesses referente a este artigo.

\section{REFERÊNCIAS BIBLIOGRÁFICAS}

1. Laur DJ, Anderson T, Geddes G, Crandall A, Pincivero DM. The effects of acute stretching on hamstring muscle fatigue and perceived exertion. J Sports Sci. 2003;21(3):163-70.

2. Zakas A, Doganis G, Papakonstandinou V, Sentelidis T, Vamvakoudis E. Acute effects of static stretching duration on isokinetic peak torque production of soccer players. J Bodywork Mov Ther. 2006;10:89-95.

3. Zakas A. The effect of stretching duration on the lower-extremity flexibility of adolescent soccer players. J Bodywork Mov Ther. 2005;9:220-5.

4. Cross KM, Worrell TW. Effects of a static stretching program on the incidence of lower extremity musculotendinous strains. J Athl Train. 1999;34(1):11-4

5. Behm DG, Bambury A, Cahill F, Power K. Effect of acute static stretching on force, balance, reaction time, and movement time. Med Sci Sports Exerc. 2004;36(8):1397-402.

6. Davis DS, Ashby PE, McCale KL, McQuain JA, Wine JM. The effectiveness of 3 stretching techniques on hamstring flexibility using consistent stretching parameters. J Strength Cond Res. 2005;19(1):27-32.

7. Kokkonen J, Nelson AG, Cornwell A. Acute muscle stretching inhibits maximal strength performance Res Q Exerc Sport. 1998;69(4):411-5.

8. Marek SM, Cramer JT, Fincher AL, Massey LL, Dangelmaier SM, Purkayastha S, et al. Acute effects of static and proprioceptive neuromuscular facilitation stretching on muscle strength and power output. J Athl Train. 2005;40(2):94-103.

9. Behm DG, Bradbury EE, Haynes AT, Hodder JN, Leonard AM, Paddock NR. Flexibility is not related to stretch-induced deficits in force or power. J Sports Sci \& Med. 2006;5:33-42
10. Brandenburg JP. Duration of stretch does not influence the degree of force loss following static stretching. J Sports Med Phys Fitness. 2006;46(4):526-34.

11. Spernoga SG, Uhl TL, Arnold BL, Gansneder BM. Duration of maintained hamstring flexibility after a one-time, modified hold-relax stretching protocol. J Athl Train. 2001;36(1):44-8.

12. Papadopoulos G, Siatras Th, Kellis S. The effect of static and dynamic stretching exercises on the maximal isokinetic strength of the knee extensors and flexors. Isokinet Exerc Sci. 2005;13(4):285-91.

13. Handel M, Horstmann T, Dickhuth H, Gulch RW. Effects of contract-relax stretching training on muscle performance in athletes. Eur J Appl Physiol Occup Physiol. 1997;76(5):400-8.

14. Achour AJ, editor. Exercícios de alongamento: anatomia e fisiologia. $2^{2}$ ed., São Paulo: Manole, 2005.

15. Stell RGD, editor. Principles and procedures of statistics. A biometrical approach. $1^{\text {a. }}$. ed., Nova York: McGraw-Hill Companies, 1997.

16. Callegari-Jacques SM, editor. Bioestatística. Princípios e Aplicações. 1ª. ed, Porto Alegre: Artmed, 2003.

17. Zakas A, Balaska P, Grammatikopoulou MG, Zakas N, Vergou A. Acute effects of stretching duration on the range of motion of elderly women. J Bodywork Mov Ther. 2005;9:270-6.

18. Mahieu NN, McNair P, De Muynick M, Stevens V, Blanckaert I, Smits N, Witvrouw E. Effect of static and ballistic stretching on the muscle-tendon tissue properties. Med Sci Sports Exerc. 2007;39(3):494-501.

19. Kotzamanidis C. Are the antagonist muscles fatigued during a fatigue task of agonist muscles? Isokinet Exerc Sci. 2004;12(3):167-71 